\title{
DWINDLABLE $R$-ALGEBRAS
}

\author{
MIROSLAV KURES̆
}

\begin{abstract}
The concept of a dwindlable $R$-algebra is defined for an arbitrary topological $R$-algebra $A$. It is proved that if $A$ is dwindlable, then its subalgebra of fixed points is trivial. It is also demonstrated that for an algebra gradable by radical its dwindlability depends on a dwindlability of its factor.
\end{abstract}

\section{INTRODUCTION}

In [5] we introduced the concept of so-called dwindlable Weil algebras. This concept should be more precise and general and this is the aim of this paper. Further, the property "to be dwindlable" implied the triviality of the fixed point subalgebra (with respect to all automorphisms) in the mentioned paper. The question is, does it hold in general? Section 1 is devoted to the recalling of basic concepts, exact definitions and the formulation of problems. We also present some original examples for a clear understanding. The main results are in Section 2. We notice that [2] studies how the dwindlability is related to the possessing of a non-trivial torus of the identity component of $\operatorname{Aut}_{\mathbb{R}} A$, where $A$ is a Weil algebra. In the survey paper [3] a number of claims concerning the form of subalgebras of fixed points of various Weil algebras are demonstrated.

\section{BASIC CONCEPTS}

\subsection{On rings and homomorphisms}

1.1.1. Polynomial rings. Let $R$ be a commutative ring. The polynomial (in one indeterminate) over a ring $R$ is defined as a map $a: \mathbb{N}_{0} \rightarrow R$ whose support is finite. Equivalently, polynomials can be defined as sequences $\left(a_{0}, a_{1}, a_{2}, \ldots\right)$ such that all but a finite number of $a_{i}$ 's are zeros. A polynomial $a: \mathbb{N}_{0} \rightarrow R, a(0)=r_{0}$, $a(1)=r_{1}, a(2)=r_{2}, \ldots$, will be denoted by $r_{0} X^{0}+r_{1} X^{1}+r_{2} X^{2}+\ldots$ (only a finite number of $r_{i}$ 's are non-zeros) with well established simplifications (we do not write terms $0_{R} X^{i}$, etc.); we also define the addition and the multiplication of polynomials in the usual way. Then polynomials form a ring denoted by $R[X]$,

$M S C$ (2010): primary 13J20, 13J30.

Keywords: topological ring, fixed point subalgebra.

The author was supported by the project FSI-S-11-3 of the Faculty of Mechanical Engineering, Brno University of Technology. 
which is a classical result. Let $R[X]_{(k: S)}=\left\{a \in R[X] ; a_{n} \in S\right.$ if $\left.n \leq k\right\}$; if $S$ is a subring of $R$, then $R[X]_{(k: S)}$ is a subring of $R[X]^{1}$.

1.1.2. $R$-algebras. Let $R$ and $A$ be commutative rings and $A$ an $R$-algebra determined by a ring homomorphism $\varphi: R \rightarrow A$. (We always consider only homomorphisms which send $1_{R}$ onto $1_{A}$.) We denote by $\hat{R} \subseteq A$ the image of $R$ by $\varphi$; evidently, $\hat{R}$ is also an $R$-algebra and $R$-subalgebra of $A$. The multiplication of elements from $A$ by elements from $R$ is in fact defined by the multiplication of elements from $A$ by elements from $\hat{R}$. If $\varphi$ is a monomorphism, then $\hat{R}$ is identified with $R$, we say that $A$ contains a copy of $R$. It comes automatically if $R$ is a field.

If we have two $R$-algebras $A_{1}$ and $A_{2}$, we have two ring homomorphisms $\varphi_{1}: R \rightarrow A_{1}, \varphi_{2}: R \rightarrow A_{2}$. A ring homomorphism $\psi: A_{1} \rightarrow A_{2}$ is called the $R$-algebra homomorphism, if $\psi \circ \varphi_{1}=\varphi_{2}$.

1.1.3. Augmentation. We will also study a possibility that there exists an $R$ algebra epimorphism $\kappa: A \rightarrow \hat{R}$. If the $R$-algebra $A$ is equipped with an $R$-algebra epimorphism $\lambda: A \rightarrow R$, we say that $A$ is an augmented $R$-algebra. Of course, for every augmented $R$-algebra there exists an $R$-algebra epimorphism $\kappa: A \rightarrow \hat{R}$ because it is given by $\kappa=\varphi \circ \lambda$.

1.1.4. Examples. Let us present the situation in examples.

Example 1.1. If $R=\mathbb{Q}$ and $A=\mathbb{Z}$, then a ring homomorphism $\varphi: R \rightarrow A$ does not exist (because $\varphi(1)=1, \varphi\left(\frac{1}{2}\right)=x \in \mathbb{Z}, x+x=\varphi\left(\frac{1}{2}\right)+\varphi\left(\frac{1}{2}\right)=$ $\varphi\left(\frac{1}{2}+\frac{1}{2}\right)=\varphi(1)=1$ has no integer solution).

Example 1.2. If $R=\mathbb{Z}[X]$ and $A=\mathbb{Z}$, then we can take the map sending a polynomial to its constant term as a ring homomorphism. However, it is not a monomorphism. So, $\mathbb{Z}$ is a $\mathbb{Z}[X]$-algebra, but $\mathbb{Z}$ does not contain a copy of $\mathbb{Z}[X]$.

Example 1.3. If $R=\mathbb{Z}$ and $A=\mathbb{Q}$, then a ring homomorphism $\varphi: R \rightarrow A$ exists $(\mathbb{Q}$ is a $\mathbb{Z}$-algebra), but a homomorphism $\kappa: A \rightarrow \hat{R}$ does not exist.

Example 1.4. If $R=\mathbb{Z}[X]$ and $A=\mathbb{Q}[X]_{(0: \mathbb{Z})}$, then we can take the map sending a polynomial with integer coefficients to the same polynomial as a ring homomorphism. So, $A=\mathbb{Q}[X]_{(0: \mathbb{Z})}$ is a $\mathbb{Z}[X]$-algebra by this homomorphism and contains a copy of $\mathbb{Z}[X]$. We also find a homomorphism $\kappa: A \rightarrow \hat{R}$ : we take a homomorphism sending a polynomial to its constant term (which is integer here), but it is impossible to take an epimorphism here.

Proof. Let $\kappa: \mathbb{Q}[X]_{(0: \mathbb{Z})} \rightarrow \mathbb{Z}[X]$ be an epimorphism. Then $\kappa(X)=y \in \mathbb{Z}[X]$ : let us denote by $u$ the leading coefficient of $y$. Then $\frac{1}{2 u} X \in \mathbb{Q}_{(0: \mathbb{Z})}$ and $y=$ $\kappa(\underbrace{\left(\frac{1}{2 u}+\cdots+\frac{1}{2 u}\right)}_{2 u \text {-times }} X)=\underbrace{\kappa\left(\frac{1}{2 u} X\right)+\cdots+\kappa\left(\frac{1}{2 u} X\right)}_{2 u \text {-times }}=\underbrace{z+\cdots+z}_{2 u \text {-times }}$ and the leading coefficient of $z$ cannot be an integer and this is a contradiction.

\footnotetext{
${ }^{1}$ This concept can be generalized by numerous ways. For instance, a sequence $S_{1} \subset \ldots S_{r} \subset R$ of subrings of $R$ can be taken and $R[X]_{\left(k_{1}: S_{1}, \ldots, k_{r}: S_{r}\right)}$ can be considered $\left(k_{1}<\cdots<k_{r}\right)$. Another possibility of generealization is to extend this concepts to polynomials of $n$ indeterminates.
} 
Example 1.5. If $R=\mathbb{Z}[X]$ and $A=\mathbb{Q}[X]_{(0: \mathbb{Z})}$, then we can take another map sending a polynomial with integer coefficients to its constant term viewed as a constant polynomial in $\mathbb{Q}_{(0: \mathbb{Z})}$. It is also a ring homomorphism and $A=\mathbb{Q}_{(0: \mathbb{Z})}$ is a $\mathbb{Z}[X]$-algebra by this homomorphism, too. $A=\mathbb{Q}[X]_{(0: \mathbb{Z})}$ does not contain a copy of $\mathbb{Z}[X]$, but there exists a homomorphism $\kappa: A \rightarrow \hat{R}$. It was proved in the previous example that an epimorphism $\lambda: \mathbb{Q}[X]_{(0: \mathbb{Z})} \rightarrow \mathbb{Z}[X]$ does not exist, so $A=\mathbb{Q}[X]_{(0: \mathbb{Z})}$ is not an augmented $\mathbb{Z}[X]$-algebra.

From now on, we assume that $A$ is an augmented $R$-algebra containing a copy of $R$. Then there exists an $R$-algebra epimorphism $\kappa: A \rightarrow \hat{R}$.

\subsection{Free $R$-algebras}

1.2.1. Modules and generator sets. Of course, an $R$-algebra $A$ is also an $R$ module. Let $\mathcal{G}=\left\{a_{i} ; i \in I\right\}$ ( $I$ is an arbitrary index set) be a set of elements of $A$. Then all elements of the form $a=\sum_{i} r_{i} a_{i}$, where $r_{i} \in R, i \in I$, represent a submodule of $A$. This submodule is denoted by $\operatorname{span}(\mathcal{G})$. Elements of $\mathcal{G}$ are called generators of $A$ if $\operatorname{span}(\mathcal{G})=A$ and then $\mathcal{G}$ is called a generator set of $A$. An $R$-module $A$ can have many generator sets, especially, $A=\operatorname{span}(A)$ always holds.

For $\mathcal{G}=\left\{a_{i} ; i \in I\right\}$, it is clear, that $a_{i_{0}} \in \operatorname{span}(\mathcal{G})$ for all $i_{0} \in I$. However, if $a_{i_{0}} \notin \operatorname{span}\left(\mathcal{G}-\left\{a_{i_{0}}\right\}\right)$ is satisfied for all $i_{0} \in I$, we say that the generator set $\mathcal{G}$ is minimal. In general, an $R$-module $A$ need not have a minimal generator set. Nevertheless, it can also have many minimal generator sets, cardinalities of them can be different.

1.2.2. Basis. Elements $\left\{a_{i} ; i \in I\right\}$ are linearly independent if, whenever $\sum_{i} r_{i} a_{i}=$ $0\left(r_{i} \in R\right)$, all $r_{i}=0$. A basis of $A$ is a linearly independent minimal set of generators of $A$. If an $R$-module $A$ has a basis, then $A$ is usually called a free $R$-module, in our case, where $A$ is an $R$-algebra, we use the name free $R$-algebra.

Remark 1.6. (IMP-rings and IBN-rings) A ring has the invariant minimality property (IMP) and is called IMP-ring if, for each minimally generated $R$-module $A$, the number of elements in each minimal generator set of $A$ is invariant, see [6]. Similarly, a ring is defined to be an invariant basis number ring or IBN-ring if for every free module, the number of elements in a basis is invariant, see [1]. The main result of [6] is that a ring $R$ has IMP if and only if $R$ is a local ring.

1.2.3. Algebra and module homomorphisms. For a clear understanding, the roman font (Hom, End, Aut, ...) is used for algebra homomorphisms and the italic font $(H o m, E n d$, Aut,$\ldots$ ) for module homomorphisms from now on.

If $A$ is a free $R$-algebra with a finite basis $a_{1}, \ldots, a_{d}$, every element of $\operatorname{End}_{R} A$ can be expressed by a matrix

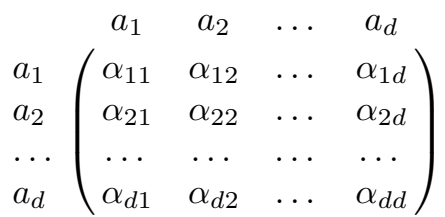


expressing that homomorphism maps $a_{1}$ onto $\alpha_{11} a_{1}+\alpha_{12} a_{2}+\cdots+\alpha_{1 d} a_{d}$, etc. Further, elements of $\operatorname{End}_{R} A$ can be expressed by a matrix in which some rows are already determined by the ring multiplication, moreover $\alpha_{11}=1, \alpha_{12}=\cdots=$ $\alpha_{1 d}=0^{2}$. Matrices of module automorphisms (elements of $A u t_{R} A$ ) form the general linear group GL( $A)$. Finally, $R$-algebra automorphisms (elements of $\operatorname{Aut}_{R} A$ ) represent a subgroup of $\mathrm{GL}(A)$. To recognize the rows of an automorphism matrix that determine automorphisms, it is useful to analyse the possibilities of an algebra gradation.

Remark 1.7. (Lie groups and algebras) If $\mathrm{GL}(A)$ is a Lie group (it comes, e.g. for $A=\mathbb{R}$ ), then the group $\operatorname{Aut}_{R} A$ of $R$-algebra automorphisms of $A$ is a closed subgroup of $\mathrm{GL}(A)$ and, therefore, $\operatorname{Aut}_{R} A$ is a Lie group, too. The Lie algebra of $\mathrm{GL}(A)$ is $\mathfrak{g l}(A)=\operatorname{End}_{R} A$ and the Lie algebra of $\operatorname{Aut}_{R} A$ is

$$
\mathfrak{a u t}_{R} A=\left\{D \in \operatorname{End}_{R} A ; \exp (t D) \in \operatorname{Aut}_{R} A \forall t \in \mathbb{R}\right\} .
$$

The algebra of derivations of $A$ is defined as

$$
\operatorname{Der}_{R} A=\left\{D \in \operatorname{End}_{R} A ; D(a b)=D(a) b+a D(b) \forall a, b \in A\right\} .
$$

In the classical Lie group theory, the identification $\operatorname{Der}_{R} A=\mathfrak{a u t}_{R} A$ is proved.

\subsection{The first observation and the formulation of a problem}

The existence of an $R$-algebra epimorphism $\kappa: A \rightarrow \hat{R}$ means that we have an ideal $\mathfrak{k}=\operatorname{ker} \kappa$ and $\kappa: A \rightarrow A / \mathfrak{k}$. The lemma below represents an elementary observation.

Lemma 1.8. The following statements are equivalent:

(i) $\kappa \in \operatorname{Aut}_{R} A$

(ii) $A=\hat{R}$

(iii) $\operatorname{ker} \kappa=\left\{0_{A}\right\}$

Proof. "(i) $\Rightarrow$ (ii)": if $\kappa \in \operatorname{Aut}_{R} A$, then $\kappa(A)=A$ and in order to $\kappa$ be an epimorphism onto $\hat{R}$ is necessary to be $A=\hat{R}$. "(ii) $\Rightarrow$ (iii)": if $\kappa: A \rightarrow \hat{R}$ is an $R$-epimorphism, then $\kappa=\operatorname{id}_{A}$ and only $0_{A}$ maps onto $0_{A}$. "(iii) $\Rightarrow$ (i)": if ker $\kappa=\left\{0_{A}\right\}$, then $\kappa$ is injective and simultaneously $\kappa$ is an $R$-epimorphism $A \rightarrow A$ : thus, $\kappa \in \operatorname{Aut}_{R} A$.

In the paper, we discuss the problem whether there are automorphisms which are "near" to $\kappa$ and what can be implied by this. Hence, we need the notion of a convergence.

\subsection{The convergence}

Definition 1.9. ([8, p. 77]) A topological ring is a ring $A$ together with a metric $\rho$ on $A$ such that ring operations are continuous with respect to the metric topology.

If $A$ is a topological ring, we can consider the pointwise convergence on the set $\operatorname{End}_{R} A$ of all $R$-algebra endomorphisms.

\footnotetext{
${ }^{2}$ When taking $a_{1}=1_{A}$.
} 
Definition 1.10. Let $\kappa: A \rightarrow \hat{R}$ be an $R$-algebra epimorphism. We say that topological $R$-algebra $A$ with a metric $\rho$ is $\rho$-dwindlable if there exist a sequence $\boldsymbol{\alpha}: \mathbb{N} \rightarrow \operatorname{Aut}_{R} A$ of $R$-algebra automorphisms ${ }^{3}$ such that $\left\{\alpha_{n}\right\}$ converges to $\kappa$ pointwise with respect to $\rho$.

We say that a topological $R$-algebra $A$ is dwindlable if there exists a metric $\rho$ in which $A$ is $\rho$-dwindlable.

Remark 1.11. It is evident that there can exist two metrics $\rho_{1}, \rho_{2}$ such that $A$ is $\rho_{1}$-dwindlable and $A$ is not $\rho_{2}$-dwindlable; in particular, if $\kappa \notin$ Aut $A$, then $A$ is not dwindlable for the discrete metric, but it can be dwindlable, e.g. for the euclidean metric ${ }^{4}$. On the other hand, for the case $\kappa \in \operatorname{Aut}_{R}(A)$, we can take the discrete metric and see that $A$ is dwindlable considering the constant sequence of automorphisms formed only by $\kappa$.

\section{Results}

\subsection{Non-trivial fixed point subalgebra}

Let $A$ be an $R$-algebra. Its fixed point subalgebra is defined by

$$
S A=\left\{a \in A ; \alpha(a)=a \forall \alpha \in \operatorname{Aut}_{R} A\right\} .
$$

Remark 2.1. Indeed, fixed points form an $R$-subalgebra. In algebraic literature, the denotation $A^{\text {Aut }_{R} A}$ for $S A$ is also used.

Definition 2.2. The fixed point subalgebra is called trivial, if $S A \subseteq \hat{R}$.

Remark 2.3. In fact, from $S A \subseteq \hat{R}$ follows that $S A=\hat{R}$. Proof. Let $\varphi: R \rightarrow$ $A$ is the ring homomorphism giving to $A$ the $R$-algebra structure, let $a \in \hat{R}$, $\alpha \in \operatorname{Aut}_{R} A, \alpha(a)=b$. As $\alpha \circ \varphi=\varphi, a=b$.

Theorem 2.4. Let $A$ be an R-algebra containing a copy $\hat{R}$ of $R, A \supset \hat{R}$, and let $\kappa: A \rightarrow \hat{R}$ be an $R$-epimorphism. If $A$ is dwindlable, then $S A$ is trivial.

Proof. Let us assume that $A$ is dwindlable and $S A$ is not trivial. Then, there exists an element $x \in A-\hat{R}$ such that $\alpha(x)=x$ for all $\alpha \in \operatorname{Aut}_{R} A$. For such an $x$, we denote by $y$ its image through $\kappa$, i.e. $\kappa(x)=y \in \hat{R}$. As $\kappa(y)=y$, we consider $z=x-y$. The element $z$ belongs to $A-\hat{R}$ (it follows from $x \neq y$ ) and $\kappa(z)=\kappa(x)-\kappa(y)=y-y=0_{A}$, so $z$ belongs also to ker $\kappa$. If $x$ is fixed and $y$ is fixed, then $z$ must be fixed, too.

There is also an infinite sequence $\left\{\alpha_{n}\right\}=\left\{\alpha_{n} \in \operatorname{Aut}_{R} A\right\}_{n=1}^{\infty}$ of $R$-algebra automorphisms such that $\left\{\alpha_{n}\right\}$ converges to $\kappa$. It implies

$$
0_{A}=\kappa(z)=\lim \alpha_{n}(z)=\lim z=z \neq 0_{A}
$$

and it is the contradiction.

\footnotetext{
${ }^{3}$ We write, as usual, $\boldsymbol{\alpha}(n)=\alpha_{n}$ and $\boldsymbol{\alpha}(\mathbb{N})=\left\{\alpha_{n}\right\}=\left\{\alpha_{n}\right\}_{n=1}^{\infty}$.

${ }^{4}$ For instance, dual numbers over reals have a form $a+b \varepsilon, a, b \in \mathbb{R}, \varepsilon^{2}=0$; automorphisms of them act by $\varphi(a+b \varepsilon)=a+C b \varepsilon, C \in \mathbb{R}-\{0\}$ and a sequence $C \rightarrow 0$ provides the dwindlability.
} 
2.1.1. Non-dwindlable algebra with the trivial fixed point subalgebra. A Weil algebra is a local commutative $\mathbb{R}$-algebra $A$ with identity, the nilradical (nilpotent ideal) $\mathfrak{n}_{A}$ of which has finite dimension as a vector space and $A / \mathfrak{n}_{A}=\mathbb{R}$. Weil algebras can be expressed as finite dimensional factors of the algebra of real polynomials in several indeterminates as follows. Let $\mathbb{R}\left[X_{1}, \ldots, X_{n}\right]$ denote the $\mathbb{R}$-algebra of real polynomials and $\mathfrak{m}=\left(X_{1}, \ldots, X_{n}\right)$ its maximal ideal. Let

$$
\mathbb{D}_{n}^{r}=\mathbb{R}\left[X_{1}, \ldots, X_{n}\right] / \mathfrak{m}^{r+1} .
$$

We denote by $\mathfrak{n}$ the nilradical of $\mathbb{D}_{n}^{r}$ and consider an ideal $\mathfrak{i}$ such that $\mathfrak{i} \subseteq \mathfrak{n}^{2}$. Then,

$$
A=\mathbb{D}_{n}^{r} / \mathrm{i} .
$$

Example 2.5. (Adopted from [5]) We present that an example of a nondwindlable Weil algebra with trivial $S A$. Let $A=\mathbb{R}[X, Y] /\left(X Y^{2}+X^{5}, X^{2} Y+\right.$ $\left.Y^{5}\right)+\mathfrak{m}^{6}$. The elements of $A$ have a form

$$
\begin{array}{rl}
k_{1}+k_{2} X+k_{3} Y+k_{4} X^{2}+k_{5} & X Y+k_{6} Y^{2}+k_{7} X^{3} \\
& +k_{8} X^{2} Y+k_{9} X Y^{2}+k_{10} Y^{3}+k_{11} X^{4}+k_{12} Y^{4}
\end{array}
$$

with the simultaneous vanishing of all monomials of the sixth or higher order in common with $X^{2} Y^{2}, X Y^{3}, X^{3} Y, X Y^{2}+X^{5}$ and $X^{2} Y+Y^{5}$. We shall describe automorphisms of $A$. The starting point for their specification is a form

$$
\begin{aligned}
\bar{X}=A X+B Y+C X^{2}+ & D X Y+E Y^{2}+F X^{3}+G X^{2} Y \\
& +H X Y^{2}+I Y^{3}+J X^{4}+K Y^{4} \\
\bar{Y}=L s+M t+N X^{2}+ & O X Y+P Y^{2}+Q X^{3}+R X^{2} Y \\
& +S X Y^{2}+T Y^{3}+U X^{4}+V Y^{4}
\end{aligned}
$$

The matrix $\left(\begin{array}{cc}A & B \\ L & M\end{array}\right)$ must be regular and we settle the conditions $\bar{X}^{2} \bar{Y}^{2}=0, \bar{X} \bar{Y}^{3}=$ $0, \bar{X}^{3} \bar{Y}=0, \bar{X} \bar{Y}^{2}+\bar{X}^{5}=0$ and $\bar{X}^{2} \bar{Y}+\bar{Y}^{5}=0$ now. The condition $\bar{X}^{2} \bar{Y}^{2}=0$ gives $A=M=0$ (Variant I) or $B=L=0$ (Variant II). The condition $\bar{X} \bar{Y}^{3}=0$ gives $C=0$ in the Variant I and $E=0$ in the Variant II. The condition $\bar{X}^{3} \bar{Y}=0$ gives $P=0$ in the Variant I and $N=0$ in the Variant II. The condition $\bar{X} \bar{Y}^{2}+\bar{X}^{5}=0$ gives $L^{2}=B^{4}, F=0$ in the Variant I and $M^{2}=A^{4}, I=0$ in the Variant II. The condition $\bar{X}^{2} \bar{Y}+\bar{Y}^{5}=0$ gives $B^{2}=L^{4}, T=0$ in the Variant I and $A^{2}=E^{2}+M^{4}$, $Q=0$ in the Variant II. Finally, we obtain $A=C=F=M=P=T=0, B$ and $L$ equal 1 or -1 in the Variant I or $B=E=I=L=N=Q=0, A$ and $M$ equal 1 or -1 in the Variant II. Hence the automorphisms have the following form

$$
\begin{aligned}
& \bar{X}=\epsilon_{1} Y+D X Y+E Y^{2}+G X^{2} Y+H X Y^{2}+I Y^{3}+J X^{4}+K Y^{4} \\
& \bar{Y}=\epsilon_{2} X+N X^{2}+O X Y+Q X^{3}+R X^{2} Y+S X Y^{2}+U X^{4}+V Y^{4}
\end{aligned}
$$

or

$$
\begin{aligned}
& \bar{X}=\epsilon_{1} X+C X^{2}+D X Y+F X^{3}+G X^{2} Y+H X Y^{2}+J X^{4}+K Y^{4} \\
& \bar{Y}=\epsilon_{2} Y+O X Y+P Y^{2}+R X^{2} Y+S X Y^{2}+T Y^{3}+U X^{4}+V Y^{4},
\end{aligned}
$$

where $\epsilon_{1}, \epsilon_{2} \in\{-1,1\}$. Evidently, $A$ is not dwindlable.

Finally, we solve the equation

$$
k_{1}+k_{2} \bar{X}+k_{3} \bar{Y}+k_{4} \bar{X}^{2}+k_{5} \bar{X} \bar{Y}+k_{6} \bar{Y}^{2}+k_{7} \bar{X}^{3}+k_{8} \bar{X}^{2} \bar{Y}
$$




$$
\begin{aligned}
& +k_{9} \bar{X} \bar{Y}^{2}+k_{10} \bar{Y}^{3}+k_{11} \bar{X}^{4}+k_{12} \bar{Y}^{4}=k_{1}+k_{2} X+k_{3} Y+k_{4} X^{2} \\
& \quad+k_{5} X Y+k_{6} Y^{2}+k_{7} X^{3}+k_{8} X^{2} Y+k_{9} X Y^{2}+k_{10} Y^{3}+k_{11} X^{4}+k_{12} Y^{4}
\end{aligned}
$$

for $k_{i}, i=1, \ldots, 12$, by use the described automorphisms. By comparing of coefficients standing at powers of $s$ and $t$, we find that $k_{2}=k_{3}=k_{4}=k_{5}=k_{6}=$ $k_{7}=k_{8}=k_{9}=k_{10}=k_{11}=k_{12}=0$ and $k_{1}$ is an arbitrary real coefficient. Thus $S A=\mathbb{R}$.

\subsection{Graded local algebras}

Let $A$ be a local algebra with the maximal ideal $\mathfrak{m}_{A}$ and let $\mathfrak{k}_{A}=\operatorname{ker} \kappa=\mathfrak{m}_{A}$. A gradation on $A$ is a family $\left(A_{i}\right)_{i \in \mathbb{Z}}$ of submodules of $A$ such that $A=\bigoplus_{i} A_{i}$ and $A_{i} A_{j} \subseteq A_{i+j}$, for all $i, j \in \mathbb{Z}$. The gradation is called by the radical ${ }^{5}$ when $A$ is isomorphic to $G(A)$, where $G(A)$ is so-called the associated graded algebra of $A$ defined by $G(A)_{i}=\mathfrak{m}_{A}^{i} / \mathfrak{m}_{A}^{i+1}$ (or equivalently, $A_{i}=0$ for $i<0$ and $\mathfrak{m}_{A}^{j}=\bigoplus_{i \geq j} A_{i}$ for each $j \geq 0$ ), see [7].

Let us suppose that $A$ is gradable by the radical. We will demonstrate that a dwindlability of $A$ depends on $A / \mathfrak{m}_{A}^{2}$ in a certain sense.

Theorem 2.6. Let $A$ be a local $R$-algebra gradable by the radical and let $B=$ $A / \mathfrak{m}_{A}^{2}$. Let $\left\{\alpha_{n}\right\}$ be a sequence of restrictions of $R$-algebra automorphisms of $A$ converging to $\kappa: B \rightarrow \hat{R}$. Then, $A$ is dwindlable.

Proof. Let $x, y \in \mathfrak{m}_{A} / \mathfrak{m}_{A}^{2}$. If $\lim \alpha_{n}(x)=0, \lim \alpha_{n}(y)=0$, then

$$
\lim \alpha_{n}(x y)=\lim \left(\alpha_{n}(x) \alpha_{n}(y)\right)=\lim \alpha_{n}(x) \lim \alpha_{n}(y)=0
$$

together with $x y=z \in \mathfrak{m}_{A}^{2} / \mathfrak{m}_{A}^{3}$. The same reasoning works for higher submodules of the gradation.

\section{Connected $A$-Jet groups}

Let $A$ be a Weil algebra and let us consider the group of its automorphisms $\operatorname{Aut}_{\mathbb{R}} A$.

Definition 3.1. The group $\operatorname{Aut}_{\mathbb{R}} A$ is called the $A$-jet group. If $\operatorname{Aut}_{\mathbb{R}} A$ is connected in the usual Euclidean topology we call it the connected A-jet group.

Remark 3.2. For the trivial ideal $\mathfrak{i}=\{0\}$, the group $\operatorname{Aut}_{\mathbb{R}} A=\operatorname{Aut}_{\mathbb{R}} \mathbb{D}_{n}^{r}=G_{n}^{r}$ is the known jet group (or differential group) which one can express by

$$
G_{n}^{r}=\operatorname{inv} J_{0}^{r}\left(\mathbb{R}^{n}, \mathbb{R}^{n}\right)_{0}
$$

(invertible $r$-jets from $\mathbb{R}^{n}$ onto $\mathbb{R}^{n}$ with the source and the target in zero).

It [4] was proved that $G_{n}^{r}$ is not connected but has two connected components. The idea of the proof is analogous as for the well known case $G_{n}^{1}=\operatorname{GL}(1, \mathbb{R})$.

The following result was also firstly mentioned in [4].

Proposition 3.3. There exist connected A-jet groups.

\footnotetext{
${ }^{5}$ I.e., by the Jacobson radical which identifies with $\mathfrak{m}_{A}$ as $A$ is local.
} 
Proof. Let us consider

$$
A=\mathbb{D}_{2}^{6} /\left(X^{3}+Y^{4}, X^{4}+Y^{5}\right) .
$$

The basis is

$$
\mathcal{B}(A)=\left\{1, X, Y, X^{2}, X Y, Y^{2}, X^{3}, X^{2} Y, X Y^{2}, Y^{3}, X^{3} Y, X^{2} Y^{2}, X Y^{3}, X^{2} Y^{3}\right\} .
$$

Aut $A$ is connected (in usual Euclidean topology):

$$
\begin{aligned}
& \text { singleton component } \\
X \mapsto \quad & X+\frac{1}{3}\left(-3 C_{1,4}+4\left(C_{2,4}+C_{2,5}\right)\right) X^{2}+C_{1,4} X Y+C_{1,6} X^{3}+C_{1,7} X^{2} Y \\
& +C_{1,8} X Y^{2}-\frac{4 C_{2,3}}{3} Y^{3}+C_{1,10} X^{3} Y+C_{1,11} X^{2} Y^{2}+C_{1,12} X Y^{3} \\
& +C_{1,13} X^{2} Y^{3}, \\
Y \quad \mapsto \quad & Y+C_{2,3} X^{2}+C_{2,4} X Y+C_{2,5} Y^{2}+C_{2,6} X^{3}+C_{2,7} X^{2} Y+C_{2,8} X Y^{2} \\
& +C_{2,9} Y^{3}+C_{2,10} X^{3} Y+C_{2,11} X^{2} Y^{2}+C_{2,12} X Y^{3}+C_{2,13} X^{2} Y^{3} .
\end{aligned}
$$

\subsection{Application: orientability of Weil contact elements}

Let $G$ be a linear algebraic group consisting of $q_{G} \in \mathbb{N}$ connected components. Every set of $p$ components of $G\left(1 \leq p \leq q_{G}\right)$ forming a subgroup of $G$ will be called a $p$-component subgroup of $G$.

Let us consider a smooth manifold $M, \operatorname{dim} M=m$. Let $n<m$. The $n$-dimensional submanifold $N$ in $M$ can be expressed (without any use of a parametrization) as

$$
\begin{array}{rl}
x^{k} & k=1, \ldots, n, \\
x^{j}=g^{j}\left(x^{k}\right) & j=n+1, \ldots, m,
\end{array}
$$

$g: N \rightarrow M$ being a local immersion. For a fixed $x=\left(x^{k}\right)$, i.e. $x=P$ (a point), we have obtained local coordinates of elements of the jet space $J_{P}^{r}(N, M)$ as

$$
\begin{aligned}
P^{k} & \\
U^{j} & =g^{j}(P) \\
Y_{k}^{j} & =\frac{\partial g^{j}}{\partial x^{k}}(P) \\
& \cdots \\
Y_{k_{1} \ldots k_{r}}^{j} & =\frac{1}{r !} \frac{\partial^{r} g^{j}}{\partial x^{k_{1}} \ldots \partial x^{k_{r}}}(P) .
\end{aligned}
$$

The elements of this jet space are called $n$-dimensional contact elements of the order $r$ determined by $N$ in its point $(P, g(P))$. If we unfix the point $P$ and its value, we write $x^{k}$ instead $P^{k}$ and $x^{j}$ instead $U^{j}$. Clearly, $\left(x^{k}, x^{j}\right)$ is nothing but $\left(x^{i}\right), i=1, \ldots, m$; contact elements have fiber bundle structure written locally as

$$
\begin{gathered}
\left(x^{i}, Y_{k}^{j}, \ldots, Y_{k_{1} \ldots k_{r}}^{j}\right) \\
\downarrow
\end{gathered}
$$


The bundle of $n$-dimensional contact elements of the order $r$ on the manifold $M$ is denoted usually by $K_{n}^{r} M$. We have

$$
K_{n}^{r} M=\operatorname{reg} T_{n}^{r} M / G_{n}^{r}
$$

$K_{n}^{r} M$ being the principal fiber bundle over $\operatorname{reg} T_{n}^{r} M$ with the structure group $G_{n}^{r}$ (regular velocities: the matrix $\left(y_{k}^{i}\right)$ has maximal rank $n$,

$$
G_{n}^{r}=\operatorname{inv} J_{0}^{r}\left(\mathbb{R}^{n}, \mathbb{R}^{n}\right)_{0}
$$

(reparametrizations)). $K_{n}^{1} M$ is also known as the bundle of Grassmannians.

Let $A$ be a Weil algebra and let $G$ be a $p$-component subgroup of its group of automorphisms $\operatorname{Aut}_{\mathbb{R}} A$. The universal contact element of the type $A[G]$ on $M$ determined by $\mathcal{V} \in \operatorname{reg} T^{A} M$ is defined as the equivalence class $[\mathcal{V}]_{G}$. The set of all such universal contact elements on a manifold $M$ is denoted by $K^{A[G]} M$.

We write shortly

$$
\begin{aligned}
& K^{A} M \text { instead of } K^{A[\operatorname{Aut} A]} M, \\
& K^{A+} M \text { instead of } K^{A\left[(\operatorname{Aut} A)^{+}\right]} M, \\
& K^{A \oplus} M \text { instead of } K^{A\left[(\operatorname{Aut} A)^{0}\right]} M .
\end{aligned}
$$

Universal contact elements belonging to these sets are called non-oriented, oriented and fully oriented, respectively.

Hence, the proof of Proposition 1 shows that we have included non-oriented contact elements in our approach.

Acknowledgement. The author thanks to Professor Ralph Kopperman from the City University of New York for his reading of the main part of this paper and useful comments.

\section{REFERENCES}

[1] C. Faith, Algebra: Rings, Modules and Categories I, Springer Verlag, 1973.

[2] M. Kureš, A note to the unipotency of the identity component of the group of algebra automorphisms, Analele Stiintifice Ale Universitatii Ovidius Constanta 15 (2007), 105-110.

[3] M. Kureš, Fixed point subalgebras of Weil algebras: from geometric to algebraic questions, in: Complex and Differential Geometry, Springer Proceedings in Mathematics, Springer, 2011, 183-192. DOI: 10.1007/978-3-642-20300-8_11

[4] M. Kureš, On the connectedness of jet groups and groups of automorphisms of Weil algebras, Journal of Applied Mathematics, Statistics and Informatics (JAMSI) 3 (2007), 209-214.

[5] M. Kureš and W. M. Mikulski, Natural operators lifting 1-forms to bundles of Weil contact elements, Irish Math. Soc. Bull. 49 (2002), 23-41.

[6] W. H. Rant, Rings whose modules require an invariant number of minimal generators, Missouri J. Math. Sci. 13 (2001), 4 pp.

[7] M. Saorín, Gradability of algebras, in: J. Abrams et al., Methods in Module Theory, Colorado Springs, 1991, Marcel Dekker, 1992.

[8] D. Yau, Lambda-Rings, World Scientific Publishing, 2010.

Miroslav Kureš, Institute of Mathematics, Brno University of Technology, Technická 2, 61669 Brno, Czech Republic

e-mail: kures@fme.vutbr.cz 
